\title{
Un estudio del monte jerezano de los años cuarenta: las declaraciones juradas de los propietarios de fincas forestales (1938-1943)
}

\author{
Juan Cabral Bustillos
}

El primer tercio del siglo $X X$ representa una época de transformaciones trascendentales para el monte de nuestro país. Es el comienzo de lo que algunos autores consideran la "mercantilización» del monte ${ }^{1}$, esto es, el período en el que el modelo de explotación económica tradicional de nuestros montes cede ante los criterios capitalistas. Este proceso de "modernización» pudo crear en los gestores de los montes públicos, y también en los propietarios privados, una visión optimista respecto de los cambios que se estaban introduciendo en la gestión del monte ${ }^{2}$ : incremento notable de la producción de corcho, resina y madera; crecimiento de la superficie destinada a pastos en un 27 por 100 e igual o superior índice de aumento de la ganadería ${ }^{3}$, etc. En consecuencia, tanto el producto forestal total como el rendimiento por hectárea se incrementaron de forma considerable.

El aumento de la productividad de los montes públicos y privados implicó una «transformación profunda en los métodos de explotación del monte " ${ }^{4}$, como consecuencia de la cual, si bien el monte alto pudo ganar algo de superficie entre 1900 y 1935, el monte bajo la disminuyó en un 12 por 100 , mientras que el matorral y los pastos la aumentaron en un 26

Vid. Groome, H.J., Historia de la política forestal en el Estado español, Agencia de Medio Ambiente, Madrid, 1990, pp. 81-90.

2 Véase Sanz Fernandez, J., "La historia contemporánea de los montes públicos españoles, 1812-1930. Notas y reflexiones (II)", en Garrabou, R., Barciela, C. y Jiménez Blanco, J.I. (eds.), Historia agraria de la España contemporánea. 3 El fin de la agricultura tradicional (1900-1960), Crítica, Barcelona, 1986, pp. 142-170.

3 Sanz Fernandez, J., op. cit., pp. 143-147.

4 Ibid., p. 146. 
por $100^{5}$. Paralelamente, el proceso roturador que siguió al de la desamortización, no se detuvo durante el período citado.

Teniendo en cuenta todo lo anterior, podemos afirmar que, junto a la adaptación a la "lógica del mercado» 6 , y pese al aumento del producto total de los montes, durante el primer tercio de nuestro siglo aquéllos continuaron su degradación progresiva: disminución de la superficie arbolada y de matorral en beneficio de los pastizales y las tierras de labor.

El proceso de modernización descrito anteriormente se vio impulsado con mayor fuerza por los gobiernos de Franco incluso antes de que concluyera la guerra. Y si hasta entonces se había intentado la maximización del producto del patrimonio forestal contando con la mediación de los «principios dasonómicos» 7 , esto es, haciéndolo compatible con los múltiples aprovechamientos del monte mediterráneo y con los intereses de las poblaciones rurales, cuyos ayuntamientos eran en gran parte los propietarios de los montes públicos excluidos de la desamortización, a partir de entonces se impondrá una política forestal que tendrá como finalidad principal la adaptación a las necesidades del mercado, satisfaciendo los intereses de los industriales del sector forestal, y desarticulando los modos de vida tradicionales de las poblaciones rurales ${ }^{8}$.

Una de las dificultades con que se encontraba la administración forestal para aplicar su política era, sin duda, el desconocimiento de la distribución de la superficie forestal y de los productos del monte, en su inmensa mayoría en manos privadas. Las estadisticas relativas al monte de propiedad privada se consideraron tan reducidas, incompletas y deficientes, que la Dirección General de Montes optó «...por no insertar nada sobre ello, mientras dichos datos no nos ofrezcan alguna garantía... ${ }^{9}$.

\footnotetext{
Ibid., p. 147.

Ibid., p. 148

Ibid., pp. 158-159.

Sirva como muestra el decreto sobre defensa de la riqueza forestal particular («Boletin Oficial del Estado" de 5 de octubre de 1938, no 97), el cual, pese a lo que pueda dar a entender su titulo, ya en el preámbulo del decreto señala su preocupación exclusiva por el déficit de "nuestra economía maderera".

9 DIRECCION GENERAL DE MONTES, 1935. La cita puede verse en GRUPO DE ESTUDIOS DE HISTORIA RURAL (GEHR), Estadísticas históricas de la producción agraria española. 1859-1935, Ministerio de Agricultura, Madrid, 1991, p. 63.
} 
Un estudio del monte jerezano de los años cuarenta: las declaraciones juradas...

En este sentido, Zapata señala que:

“...la administración española, que se dedicó con un tesón y eficacia encomiables a la cuantificación de los productos del patrimonio forestal público, no se molestó en averiguar el volumen de los montes y dehesas de propiedad privada ni lo que se obtenía de ellos" ${ }^{10}$.

Este desconocimiento de la situación del monte privado explica que el gobierno, a través del Servicio Nacional de Montes, solicite a los distritos forestales -entre ellos el de Cádiz- información detallada de los propietarios de fincas forestales, la distribución de las mismas en zonas arboladas, matorral, pastos y labor, los productos del monte, etc. Se trata de las "declaraciones juradas", que vamos a estudiar a continuación, ciñéndonos al caso jerezano.

\section{LAS DECLARACIONES FORESTALES DE LOS PROPIETARIOS JEREZANOS}

En agosto de 1938, la Jefatura del Distrito Forestal gaditano publicaba una circular ${ }^{11}$ en cumplimiento de una disposición del Servicio Nacional de Montes, Caza y Pesca, para que los diversos distritos forestales provinciales investigaran "todo cuanto afecte a la producción, transformación y comercio de productos forestales" de los propietarios privados que realizaran algún aprovechamiento "de madera, corcho, bellota, piña, etc.".

La circular instaba a los Ayuntamientos gaditanos a cumplir la normativa y a que colaborasen activamente en la difusión de la circular entre los propietarios y en la recogida de las declaraciones juradas de los mismos, para girarlas posteriormente a la Jefatura del Distrito Forestal, situada en la capital de la provincia.

Esta circular marca el inicio de una colaboración entre el Ayuntamiento de Jerez y el servicio forestal provincial, cuya documentación se encuentra en el legajo 1.119 de la sección de Policía Rural (Agricultura) del Archivo Municipal de Jerez. Esta colaboración, que en principio estaba previsto que durase poco más de quince dias ${ }^{12}$, se prolongó durante un periodo

\footnotetext{
10 Zapata Blanco, S., "El alcomoque y el corcho en España, 1850-1935", en Garrabou, R., Barciela, C. Y Jimenez Blanco, J.l. (eds.), op. cit., p. 232.

11 Boletín Oficial de la Provincia de Cádiz, $n^{\circ} 1.163$, de 24 de agosto de 1938.

12 En circular de la Jefatura del Distrito Forestal de Cádiz dirigida al Ayuntamiento jerezano de fecha 27 de septiembre de 1938 se comunica a esta institución el "atraso" en la
} 
mayor de dos años y, todavía en agosto de 1940, el Ayuntamiento jerezano recibe circulares en las que se amenaza con sanciones a los propietarios infractores ${ }^{13}$.

Como vemos, el destase entre lo previsto por el servicio forestal gaditano para recoger las declaraciones de los propietarios de fincas forestales y el tiempo real transcurrido es patente. Ello puede darnos una idea de la resistencia de los propietarios para hacer efectivas tales declaraciones, pero no invalida su importancia como fuente documental, puesto que pocos propietarios de fincas forestales pudieron escapar a la entrega de sus respectivas declaraciones juradas: en octubre de 1940, sólo catorce propietarios no han entregado su declaración ${ }^{14}$.

El citado legajo contiene un total de 129 declaraciones juradas, aunque nosotros sólo vamos a considerar 128 , pues una se encuentra repetida ${ }^{15}$. Estas fincas tienen una cabida de más de 55.000 hectáreas. Podemos considerar, pues, que la fuente documental que estamos comentando es de un alto grado de fiabilidad, al menos en lo que a estos datos - número de propietarios, superficie forestal- se refiere. Para hacernos una idea de su importancia, hemos de tener en cuenta que la superficie forestal jerezana no superaba en 1985 las 35.000 hectáreas ${ }^{16}$.

Las declaraciones forestales constituyen, desde nuestro punto de vista, un instrumento de primera magnitud para conocer el estado del monte jerezano en los años finales de la guerra civil y en los primeros de la postguerra, así como un punto de partida indispensable para conocer su evolución hasta la actualidad, sobre todo con los efectos que para el monte gaditano en general, y para el jerezano en particular,

entrega de las declaraciones de los propietarios de fincas forestales de Jerez y se da un plazo máximo de quince días en la recogida de datos. Asimismo, la circular recuerda que se consideran "fincas de carácter forestal incluso las que careciendo por completo de arbolado se dedican a pastos, asi como los terrenos improductivos, las marismas, etc.". ARCHIVO MUNICIPAL DE JEREZ DE LA FRONTERA (en adelante, AMJF), Policia Rural, Leg. 1.119, Declaraciones forestales.

13 Circular de la Jefatura del Distrito Forestal gaditano dirigida al ayuntamiento de Jerez de 14 de agosto de 1940. AMJF, Leg. 1119, Declaraciones...

${ }_{14}$ Ibid., Circular de la Jefatura del Distrito Forestal de Cádiz al ayuntamiento de Jerez de 21 de octubre de 1940 .

\$5 Las declaraciones juradas 55 y 96 corresponden a la finca "Pasada Blanca y Benalup", de los hermanos Ferrer, con una cabida de 791 ha.

16 En 1985, la superficie forestal jerezana era de 34.431 ha., obtenidas a partir de la suma de la superficie forestal propiamente dicha $-9.323 \mathrm{ha} .-$, el matorral - 18.014 ha. - y el pastizal 7.094 ha.-. Vid. MINISTERIO DE AGRICULTURA, PESCA Y ALIMENTACION (DIRECCION 
tuvo la legislación agraria del franquismo y de los primeros años de la transición política - leyes de fincas manifiestamente mejorables, Reforma Agraria andaluza, etc. - en el contexto de la crisis definitiva de la agricultura tradicional en España ${ }^{17}$. Con este trabajo no pretendemos, obviamente, un objetivo tan ambicioso. El objeto del presente trabajo, que señalamos a continuación, es el siguiente: 1) aproximarnos a las magnitudes globales del monte jerezano -la superficie forestal, la propiedad y su distribución-; 2) el conocimiento de las especies forestales dominantes en las fincas de este término municipal; 3) los aprovechamientos forestales más importantes según las declaraciones de los propietarios; y 4) una valoración crítica de los datos que aportan los propietarios jerezanos de fincas forestales y una reflexión final acerca de la situación peculiar del monte gaditano de la época.

\section{EL MONTE JEREZANO EN 1940}

\section{A) La superficie forestal de Jerez}

En el modelo de declaración se solicita a los propietarios que indiquen el nombre de la finca y los límites de la misma, así como la superficie total en hectáreas - algunos propietarios la expresan en aranzadas-, y las superficies arbolada, de matorral, de pastos y de cultivo de la finca. Seguidamente, la declaración demanda información sobre la especie forestal dominante en la explotación, el principal uso a que se destina y los diversos rendimientos de la misma.

Pese a la claridad que desde nuestra óptica puede observarse en el modelo de declaración jurada, los resultados de las mismas no siempre resultan plenamente satisfactorios. A lo largo de este trabajo iremos comentando las luces y la sombras de las declaraciones forestales. Ahora, lo que nos interesa destacar es que, de todos los datos, el más fiable es, sin duda, el de la superficie forestal total: unas 55.000 hectáreas, cuya distribución podemos observar en el cuadro 1 :

GENERAL DE LA PRODUCCION AGRARIA), Mapa de cultivos y aprovechamientos de la provincia de Cádiz, Madrid, 1985, p. 75.

17 Un estudio reciente de la deforestación en la provincia de Cádiz puede verse en CABRAL BustILLOS, J., "Transformaciones agrarias e incendios forestales: un indice de la deforestación en la provincia de Cádiz (1978-1989)", en Agricultura y Sociedad, núm. 59, abril-junio, 1991, Madrid, pp. 119-157. 
CUADRO 1

Superficie forestal del término de Jerez, 1938-1942*

Tipos de Superficie

Superficie (ha.)

Arbolada

22.593

Matorral

10.063

Pastos

11.458

Labor

8.641

Sin especificar

2.714

TOTAL

55.469

NotA. - El cuadro ha sido elaborado a partir de las respectivas superficies parciales - arbolada, matorral, etc.-declaradas. Algunos propietarios no la especifican. La diferencia observada en la superficie total con el cuadro 3 es insignificante.

Fuente: AMJF, Leg. 1119, Declaraciones forestales.

El modelo oficial de declaración jurada solicita de los propietarios la superficie catastral, la escriturada y la resultante de la medición de la finca. En algunos casos no coinciden ninguna de ellas, aunque esto es excepcional. En general hemos tenido en cuenta la superficie catastral, la más empleada en las declaraciones, si bien siempre hemos tenido en cuenta que el resultado de las sumas parciales - arbolada, matorral, pastos y labor - coincidiera con aquélla. Cuando no había coincidencia, hemos optado por la que más se aproximaba a la suma de los distintos tipos de superficie.

Una categoría que nos hemos visto obligados a introducir en el cuadro 1 es la de los terrenos sin especificar. Los propietarios de éstos indican la superficie total de la finca, pero no su distribución. Podemos deducir que buena parte de esos terrenos, por los aprovechamientos declarados, son de carácter forestal o arbolado, pero hemos preferido reflejar de este modo el estado de la cuestión, que, de todas maneras, no es muy significativa: menos del 5 por 100 del total ${ }^{18}$.

Los terrenos arbolados predominan con un 40,7 por 100 de la superficie forestal. Cabe interpretar que, pese a las denuncias que sobre el estado del arbolado revelan los testimonios de numerosas fuentes ${ }^{19}$,

is Asi, nos encontramos con fincas de 238 ha. con el alcornoque como especie principal, y otras de 471, 400, 350 y 326 ha., cuyas especies dominantes son el alcornoque, el quejigo y el acebuche. Son un total de diez fincas las que no declaran la superficie arbolada.

19 Las denuncias sobre el pésimo estado del arbolado son una constante a lo largo de nuestra historia moderna y contemporánea. Intormación sobre el tema abunda en el AMJF. Un ejemplo 
y de algún modo también los propios propietarios, cuando mencionan en sus declaraciones la "escasez" del arbolado, aquél mantiene un porcentaje muy importante, sobre todo si lo comparamos con el actual.

Los terrenos de matorral y pastos constituyen otras categorías difíciles de precisar. De hecho, numerosos propietarios indican conjuntamente ambas superficies. Estas forman el otro bloque importante del territorio forestal jerezano: suman algo más de 21.000 hectáreas, representando al 38,7 por 100 del total.

Los terrenos destinados a cultivo en las fincas, aunque ocupan un porcentaje menor, el 15,5 por 100 , constituyen un dato muy indicativo de que estos terrenos -las dehesas- proporcionan unos aprovechamientos múltiples, pero también un indicio del estado de la degradación progresiva del arbolado hacia el matorral y los pastos, y de éstos hacia los terrenos de labor, especialmente en las zonas llanas y de monte bajo próximas a la campiña.

En resumen: la superficie arbolada y los terrenos de matorral y pastos predominan de forma manifiesta en las fincas forestales jerezanas, que también cuentan con un porcentaje significativo de terrenos de labor.

Indicábamos más arriba que las Declaraciones forestales nos parecian bastante fiables especialmente en lo que se refiere a los datos de la superficie total. Ello viene confirmado por el hecho de que las fincas pertenecientes al Ayuntamiento jerezano también están contenidas en el legajo 1.119, y cuya relación con las fincas privadas mostramos en el cuadro 2:

puede ser el informe del guarda mayor de los montes de propios de Jerez, que en 1843 entrega un informe sobre el "notable daño y deterioro" que sufren las dehesas jerezanas a causa de la tala y el carboneo abusivos (AMJF, Leg. 139, expediente 4.041). También el "Diccionario" de Pascual Madoz nos habla de la "decadencia" y "escasez" del arbolado de Jerez, aun cuando hubo "un tiempo que gran parte de este territorio estaba poblado de infinitos árboles de toda especie". Madoz, P.. Diccionario Geográfico-Estadístico-Histórico de España y sus Posesiones de Ultramar, 1845-1850, Cádiz, edición facsímil, Ambito, Valladolid, 1986, p. 248. Por su parte. Salvador Cerón, ingeniero de montes, cita otra causa, la extracción de curtientes para la industria del cuero, en la segunda mitad del XIX. Cerón afirma que entre 1860 y 1879 "han sido cortados a mata rasa $24.850 \mathrm{Ha}$. de monte alcornocal de propiedad particular, con 1.024 .000 pies...". Cerón, S., Industria Forestal Agrícola, Cádiz, 1879. La cita puede verse en CebALLOS, L. y Martin Bolanos, M. Estudio sobre la vegetación forestal de la provincia de Cádiz, Madrid, 1930, p. 123. En el mismo sentido se expresa Zapata Vid. Zafata Blanco, S., op. cit., pp. 230279 . 


\section{CUADRO 2}

Distribución del monte entre propiedad agrícola privada y Ayuntamiento

\begin{tabular}{lrrr}
\hline Tipo de propietario & № de fincas & Superficie (ha.) & $\%$ \\
\hline Fincas privadas & 123 & 47.155 & 85 \\
Fincas del Ayuntamiento & 128 & 8.314 & 15 \\
\hline TOTAL & 128 & 55.469 & 100 \\
\hline
\end{tabular}

Fuente: AMJF, Leg. 1.119, Declaraciones...

La participación del Ayuntamiento jerezano en la propiedad forestal con un porcentaje significativo se debe a que pudo conservar una parte de los montes del caudal de propios, con una superficie próxima a las 7.000 hectáreas. El resto de los terrenos municipales son escasamente significativos, constituyendo pastizales y marismas. Ello quiere decir que, pese a la relativa importancia de la propiedad municipal, con la excepción de los montes de propios, las fincas forestales de mayor calidad se encuentran en manos privadas.

\section{B) Dimensiones de la explotación forestal jerezana}

Para facilitar el estudio de la distribución de la propiedad y de las dimensiones de la explotación forestal jerezana, hemos elaborado el cuadro 3 , en el que hemos establecido la correspondiente división en frecuencias, y señalando el número de casos de cada frecuencia, la superficie que representa, su porcentaje, y la superficie media de cada serie.

CUADRO 3

Distribución de la propiedad por intervalos

\begin{tabular}{lrrrr}
\hline Superficie (ha.) & № de casos & Superficie total (ha.) & \multicolumn{2}{c}{$\%$ Promedio } \\
\hline $0-49$ & 8 & 106 & 0,20 & 13,2 \\
$50-99$ & 21 & 1.561 & 2,80 & 74,3 \\
$100-499$ & 58 & 15.338 & 27,50 & 264,4 \\
$500-999$ & 35 & 24.4844 & 3,80 & 699,5 \\
$1.000-4.999$ & 4 & 7.431 & 13,30 & $1.857,7$ \\
$>5.000$ & 1 & 6.902 & 12,40 & $6.902,2$ \\
\hline TOTALES & $127^{*}$ & 55.822 & 100,00 & 439,5 \\
\hline
\end{tabular}

* En la elaboración del cuadro hemos tenido en cuenta la superficie total declarada de las fincas. Un propietario no especificó tal superficie, de ahi que la suma resultante sea de 127.

Fuente: AMJF, Leg. 1119, Declaraciones... 
Llama la atención la elevada superficie media de las fincas forestales jerezanas: 439,5 hectáreas. Entendemos que este promedio no se encuentra distorsionado por los valores extremos, si tenemos en cuenta que las fincas de extensión inferior a las 100 hectáreas no llegan al 3 por $100 \mathrm{del}$ total y, aunque las fincas mayores de 1.000 hectáreas tienen cierta importancia -el 25 por 100-, el bloque más numeroso está representado por el de las fincas comprendidas entre 100 y 999 ha. - 93 casos y 39.822 ha.-, que suponen el 71 por 100 del total. Los valores promedio de estas dos frecuencias, 264,4 y 699,5 hectáreas, señalan la tendencia del tamaño medio de la explotación forestal jerezana.

Como puede apreciarse, la pequeña y mediana propiedad es prácticamente inexistente ${ }^{20}$, pudiéndose afirmar que la propiedad del monte jerezano se encuentra repartida entre muy pocas familias de la élite local. Esta situación viene reforzada por el hecho de que son numerosos los nombres de familias que se repiten en las declaraciones juradas. Este es el caso de la familia Guerrero, con cuatro fincas y más de 4.500 hectáreas declaradas; los hermanos Ferrer, también con cuatro fincas y más de $2.000^{21}$; Belmonte, con más de 1.800; etc.

\section{C) La superficie arbolada}

Según las declaraciones juradas, la superficie arbolada del territorio forestal jerezano supone el 40,7 por 100 del total. Un dato de interés que puede servirnos como una primera aproximación al estudio de aquella es el análisis de los datos contenidos en la parte de las declaraciones que solicita información sobre la "especie dominante» en la finca. Es una estimación subjetiva, obviamente, pero que puede ofrecernos una impresión de las especies forestales más representativas del término de Jerez:

20 Zapata señala, citando a Vieira, que "no basta disponer de las condiciones de suelo y clima favorables al desenvolvimiento del alcornoque, para que su cultivo sea viable... Bajo valor unitario del suelo, por inadaptación a cultivos más ricos, población rural escasa y sobria y propiedades de grandes extensiones constituyen los principales requisitos para la expansión e, incluso, para la simple supervivencia del alcornocal...". ViEiRA, J., Subericultura, Ministerio de Economía, Porto, 1950. Existe una reciente edición española de esta obra coordinada por Pablo Campos Palacin; su referencia bibliogrática es la siguiente: VIEIRA, J., Subericultura, Ministerio de Agricultura, Pesca y Alimentación, Madrid, 1991. La cita, en Zapata Blanco, S., op. cit., p. 250.

21 El origen catalán de algunos propietarios es evidente. Tal es el caso de los hermanos Ferrer y de su administrador, José Ametller, industrial corchero catalán que poseía fábricas de tapones y cuadradillos de corcho "en la jurisdicción de la Cámara de Comercio de Sevilla". R. Medir Jofra señala la fecha del año 1929 para este negocio. La referencia, en MEDIR JOFRA, R., Historia del gremio corchero, ed. Alhambra, Madrid, 1953, p. 474. 
Especie dominante № de fincas

Alcornoque

Acebuche

Encina

Quejigo

Eucalipto

Pino

Otras

TOTAL

La especie forestal dominante, por emplear el termino citado en las declaraciones juradas, es el alcornoque, seguida del acebuche, la encina y el quejigo, especies del bosque mediterráneo, con 89 casos de 128 . La importancia de es tas especies es mayor aún si tenemos en cuenta la superficie real que ocupan y no solo el número de fincas. En cuanto abordemos este estudio, especies como el pino y el eucalipto quedan aun más empequeñecidas (cuadro 4).

\section{CUADRO 4}

Las especies forestales jerezanas y sus asociaciones

1. Una sola especie declarada

\begin{tabular}{l|r|r}
\hline & № de casos & Superficie (ha.) \\
\hline Alcornoque & 10 & 979 \\
Acebuche & 2 & 275 \\
Encina & 5 & 575 \\
Quejigo & 1 & 15 \\
Pino & 2 & 96 \\
Eucalipto & 7 & 20 \\
Suma parcial & 27 & 1.960 \\
\hline 2. Asociaciones del alcornoque & & \\
\hline Alcornoque, acebuche y quejigo & 14 & 12.509 \\
Alcornoque y quejigo & 8 & 2.454 \\
Alcornoque y encina & 4 & 620 \\
Alcornoque y pino & 3 & 313 \\
Alcornoque y acebuche & 3 & 200 \\
Alcornoque, encina y acebuche & 1 & 170 \\
Alcornoque y otras especies & 1 & 230 \\
Suma parcial & 34 & 16.496 \\
\hline
\end{tabular}


Un estudio del monte jerezano de los años cuarenta: las declaraciones juradas..

CUADRO 4 (continuación)

Las especies forestales jerezanas y sus asociaciones

3. Asociaciones del acebuche y la encina

\begin{tabular}{l|r|r} 
& № de casos & Superficie (ha.) \\
\hline Acebuche y encina & 6 & 1.670 \\
Acebuche y quejigo & 7 & 808 \\
Acebuche, encina y algarrobo & 2 & 161 \\
Acebuche, encina y quejigo & 1 & 120 \\
Encina y algarrobo & 2 & 750 \\
Encina y quejigo & 2 & 177 \\
Acebuche y otras especies & 1 & 5 \\
Suma parcial & 21 & 3.691 \\
\hline
\end{tabular}

4. Otras asociaciones, otras especies

\begin{tabular}{l|r|r}
\hline Olivo, etc. & 6 & 446 \\
\hline SUMA TOTAL & 88 & 22.593 \\
\hline
\end{tabular}

Fuente: AMJF, Leg. 1.119, Declaraciones...

Para el estudio de la superficie arbolada jerezana, dada la escasa importancia que tienen las fincas con una sola especie, y para ofrecer un estado de la riqueza de la vegetación forestal de su término municipal, hemos considerado oportuno crear los cuatro bloques que ofrecemos en el cuadro anterior ${ }^{22}$ : una única especie declarada, las diversas asociaciones

22 En su estudio sobre la vegetación forestal de la provincia de Cádiz, Ceballos y Martín Bolaños señalan las siguientes cifras para el término de Jerez hacia 1930 :

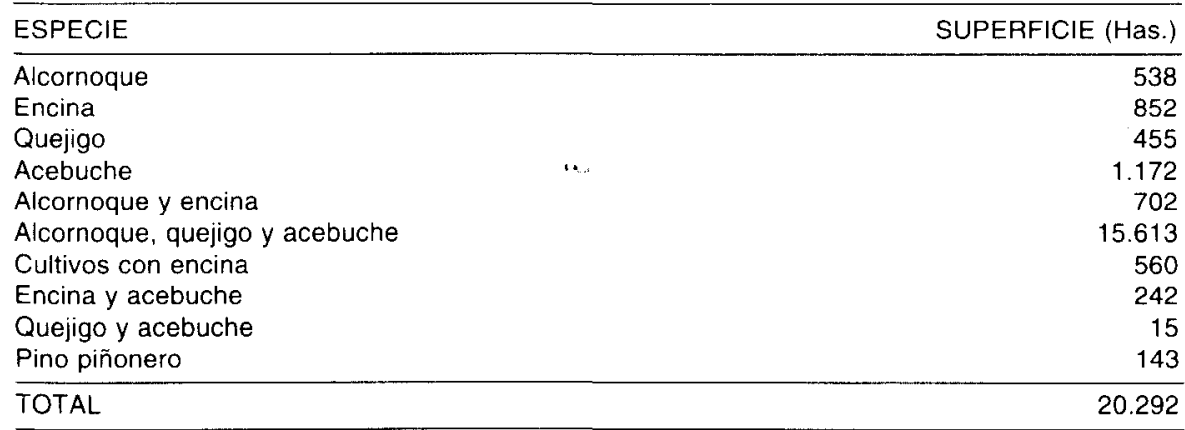

Vid. Ceballos, L, y Martin Bolaños, M., op. cit., pp. 342-343. Como vemos, la consonancia entre el cuadro 4 y estas cifras es clara, especialmente en la superficie arbolada total y en la principal asociación del alcornoque con el quejigo y el acebuche. 
del alcornoque con otras especies, las del acebuche y la encina, y otras asociaciones de escasa significación.

El cuadro 4 nos da una idea de la extraordinaria complejidad de la vegetación forestal jerezana. La especie dominante, el alcornoque, se asocia de diversas formas con el resto de las especies forestales, especialmente con el acebuche y el quejigo, asociación que representa más del 55 por 100 del total de las zonas arbóreas. En conjunto, lo que hemos denominado asociaciones del alcornoque representan el 73 por 100 de la superficie arbolada. Las asociaciones del acebuche y la encina con especies en las que no entra el alcornoque es menor - 16,3 por 100 -, pero ello no debe restar importancia a estas especies, puesto que se encuentran asociadas a su vez con el alcornoque. Tras el alcornoque, el acebuche, el quejigo y la encina, probablemente por este orden, dominan la vegetación forestal de Jerez. Como puede apreciarse, especies como el pino o el eucalipto, sobre todo este último, juegan un papel marginal en el monte jerezano.

\section{LOS APROVECHAMIENTOS DEL MONTE JEREZANO}

En esta aproximación a la economía del monte jerezano contamos con varias dificultades. La primera deriva de la extraordinaria variedad de aprovechamientos típica del monte mediterráneo: corcho, bellota, pastos, leña y carbón, madera, curtientes, etc. La segunda dificultad procede de la propia complejidad que encierran las declaraciones forestales. Recurrir a este procedimiento - la solicitud de declaraciones juradas - evidencia de modo elocuente el desconocimiento de la administración forestal acerca de lo que sucedia en unos montes mayoritariamente en manos privadas y sin sujección alguna a planes administrativos de ordenación o de control de la masa forestal.

Una tercera dificultad la constituyen las unidades de medida citadas por los propietarios. Si exceptuamos el caso del corcho, donde los propietarios declaran la producción en quintales -quintal métrico y quintal castellano de $46 \mathrm{~kg}$.- en los demás aprovechamientos, tales unidades no siempre son expresadas en términos convertibles. Así, la producción de leña y madera se refleja en quintales, toneladas y estéreos, y algunos citan una producción de madera de «200 arados», de un número determinado de "cargas", etc. Por su parte, aquéllos que tienen arrendadas las fincas expresan la renta en pesetas.

A todo lo expuesto debemos añadir la presumible ocultación de datos de los propietarios en lo que a los rendimientos de las fincas se refiere. 
Medir Jofra, citado por Zapata, afirma que todas "las cuestiones del corcho se trataron, años atrás, sobre la base de estadísticas y datos falsos" y que «ni en nuestro país ni en el vecino [Portugal] se sabian las cifras exactas de la producción forestal» ${ }^{23}$.

En la figura 1 mostramos una panorámica de los aprovechamientos del monte y las dehesas jerezanas a partir de las respuestas de los propietarios en las "declaraciones», expresados en porcentajes.

FIGURA 1

Los aprovechamientos del monte jerazano

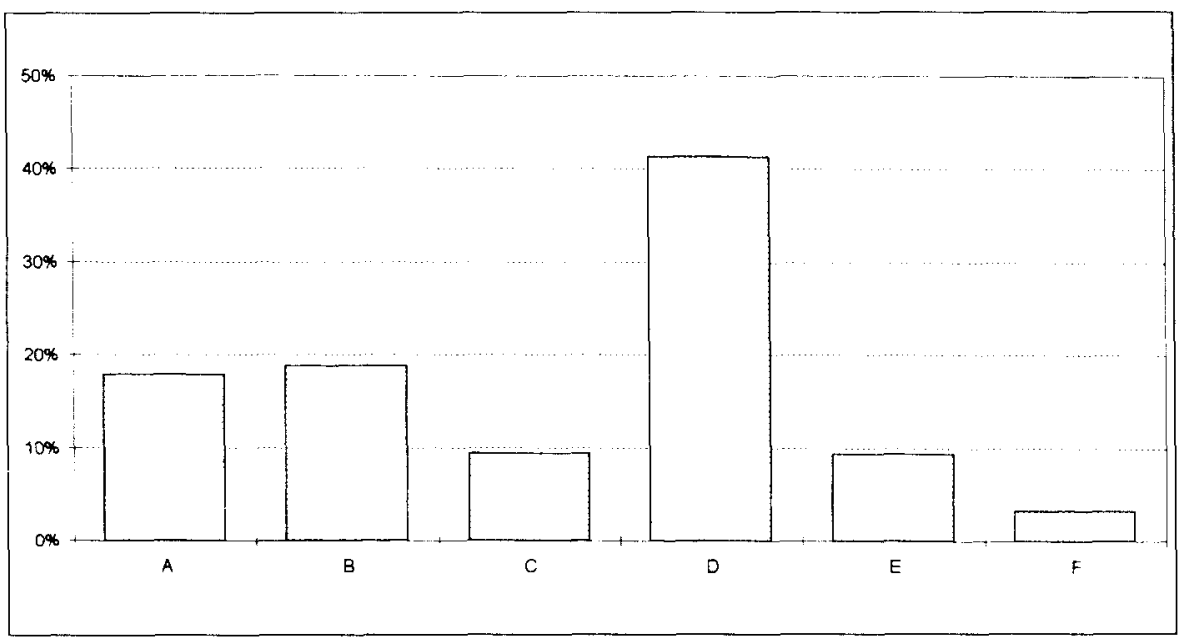

A: Corcho; B: Leña y carbón; C: Madera; D: Montanera y pastos; E: Labor; F: Otros. Fuente: ANJF, Leg. 1.119, Declaraciones...

Los propietarios mencionan los aprovechamientos del corcho en 37 ocasiones -18 por 100-y 42 fincas declaran su producción suberosa; la leña y el carbón son citadas en 39 casos - 19 por 100-; la madera -9 por 100 de las fincas-y las tierras de labor —otro 9 por 100 -. A destacar el elevado número de respuestas sobre los aprovechamientos de los pastos y la montanera-88 casos, 41 por 100 -

Aunque expresados en porcentajes, estos datos muestran la tendencia de los principales aprovechamientos que ofrece el monte jerezano: la

23 Medir Jofra, R., op. cit, p. p. 438. La cita puede verse también en Zapata Balnco, S., op. cit., p. 232. 
dehesa, donde los pastos y la montanera adquieren una gran presencia, dándonos una idea de la importante carga ganadera que soportan. Por desgracia, son muy contados los propietarios que declaran el número de cabezas de ganado vacuno, porcino, lanar, cabrio o caballar, constituyendo tal vez la principal sombra de las declaraciones juradas.

\section{A) La producción de corcho}

El aprovechamiento que mejor puede estudiarse a partir de las declaraciones juradas es el del corcho. En este sentido, cabe destacar a la provincia de Cádiz como la principal abastecedora de esta materia prima ${ }^{24}$. El número de fincas que declaran su producción suberícola es de 42 , con 234.037 quintales métricos producidos en turnos de nueve años, en una superficie de 16.344 hectáreas, lo que nos da un rendimiento medio de 14,3 quintales por hectárea. En el cuadro 5 mostramos únicamente la producción total de 36 fincas, que son las que tienen todos los datos declarados - superficie y producción de corcho-.

\section{CUADRO 5}

Distribución de la producción de corcho por intervalos

\begin{tabular}{lrrrrr}
\hline Intervalos & Producción & Promedio & Superficie & Superficie & $\begin{array}{r}\text { № de } \\
\text { casos }\end{array}$ \\
\hline $0-9,99$ & 21.336 & 5 & 4.147 & 276 & 15 \\
$10-24,99$ & 151.232 & 14 & 11.059 & 851 & 13 \\
$25-49,99$ & 37.260 & 34 & 1.093 & 219 & 5 \\
$50-99,99$ & 1.548 & 74 & 21 & 11 & 2 \\
$>100$ & 9.000 & 360 & 25 & 25 & 1 \\
\hline TOTAL & 220.376 & 13 & 16.344 & 454 & 36 \\
\hline
\end{tabular}

NOTA: La producción total, para un ciclo de nueve años. Unicamente hemos multiplicado por nueve la producción de los montes de propios, que la señalaba anualmente. Fuente: AMJF, Leg. 1.119, Declaraciones...

24 "La provincia más destacada en cuanto a cantidad de materia prima es la provincia de Cádiz". Vid. MEDIR JofRA, R., op. cit., p. 85. Para 1933, y teniendo en cuenta sólo la producción de corcho segundero y bornizo, la provincia gaditana ocupaba el primer lugar nacional, con una cuota superior al 30 por 100 . Vid. ZAPATA BLANCO, S., op. cit., p. 234. La misma conclusión podemos sacar si tenemos en cuenta la producción de los montes de utilidad pública, estudiada por el Grupo de Estudios de Historia Rural (GEHR). En 1932, los montes gaditanos de utilidad pública alcanzan una producción de "corchos y cortezas" con un valor de 548.000 pesetas, frente a las 780.000 del total nacional -el 70 por 100-. Estas cifras pueden verse en GEHR, op. cit., pp. 349 y 1.199. 
La conclusión que podemos extraer del cuadro 5 parece clara: la mayoría de los rendimientos declarados se encuentran por debajo de los 34 quintales por hectárea, y el 78 por 100 de la producción declarada, por debajo de los 14 quintales por hectárea. El promedio es, pues, de 1,5 quintales por hectárea y año. Para los años 30 , Ceballos y Martín Bolaños estiman unos rendimientos mínimos en torno a los 2 quintales por hectárea ${ }^{25}$. Aceptando estos rendimientos como referencia, tenemos que la media de los alcornocales jerezanos se encuentra un 25 por 100 por debajo de la media de la zona gaditana. Sólo catorce de las 36 fincas jerezanas declaran un rendimiento medio igual o superior a los 2 quintales por hectárea y año, sumando una producción de 94.162 quintales -más del 40 por 100 del total- en una superficie de 3.275 hectáreas -el 20 por 100 de la superficie total-.

En el conjunto provincial, la producción total de corcho de los montes de Jerez estaria entre el 21 y el 25 por $100^{26}$.

La impresión que nos ofrece el cuadro 5 es que las fincas de mayores dimensiones declaran unos rendimientos menores, y al revés, las fincas de menores dimensiones declaran unos rendimientos más elevados. Esta impresión podemos corroborarla en la figura 2 , en la que conscientemente hemos excluido la producción de los montes de propios, y que ofrece, por tanto, la relación entre la dimensión media de las fincas privadas y su producción en quintales por hectárea:

La superficie y la producción de los montes de propios han sido excluidos de su serie - la que indica una producción total mayor- para no distorsionar los resultados, dada su gran superficie- cerca de 7.000 hectáreas-y su elevada producción -75.600 quintales a razón de 8.400 por año-. De este modo, podemos comprobar cómo los montes privados de mayores dimensiones tienden a declarar unos menores rendimientos por hectárea, mientras que las fincas más pequeñas declaran unos rendimientos más

25 "De las sierras de Tarifa al Aljibe, puede suponerse una producción media total de 100.000 $\mathrm{Qm}$. anuales, lo que corresponde aproximadamente a $2 \mathrm{Qm}$. por Ha., con valor sobre el árbol en 1930 de unas 40 pesetas; en los mejores sitios de este núcleo puede liegarse a producciones de $8 \mathrm{Qm}$. y calidades cotizables a 55 pesetas. Debe observarse, al juzgar estas cifras, que el corcho ha atravesado una grave crisis desde la guerra europea hasta hace dos años." Vid. Cebal.Los, L. y Martín Bolaños, M., op. cit., p. 133.

26 La estimación sobre la producción provincial de corcho ofrecida por Ceballos y Martín Bolaños nos parece baja. Zapata, recogiendo los datos del Anuario Estadistico de España de los años 1932-33, ofrece una producción de 106.688 Qm. para 1932 y 117.972 para 1933. La producción se refiere al corcho segundero y bornizo. Y ello sin contar con el 20-30 por 100 de ocultación que sospecha Medir Jofra. Vid. ZAPATA BLANCO, S., op. cit, p. 234. La oscilación en el porcentaje que damos para la producción de corcho en los montes jerezanos tiene en cuenta el mínimo expresado por Ceballos y Martín Bolaños y el máximo de Zapata. 


\section{FIGURA 2}

Producción de corcho en relación con la superficie media de las fincas privadas

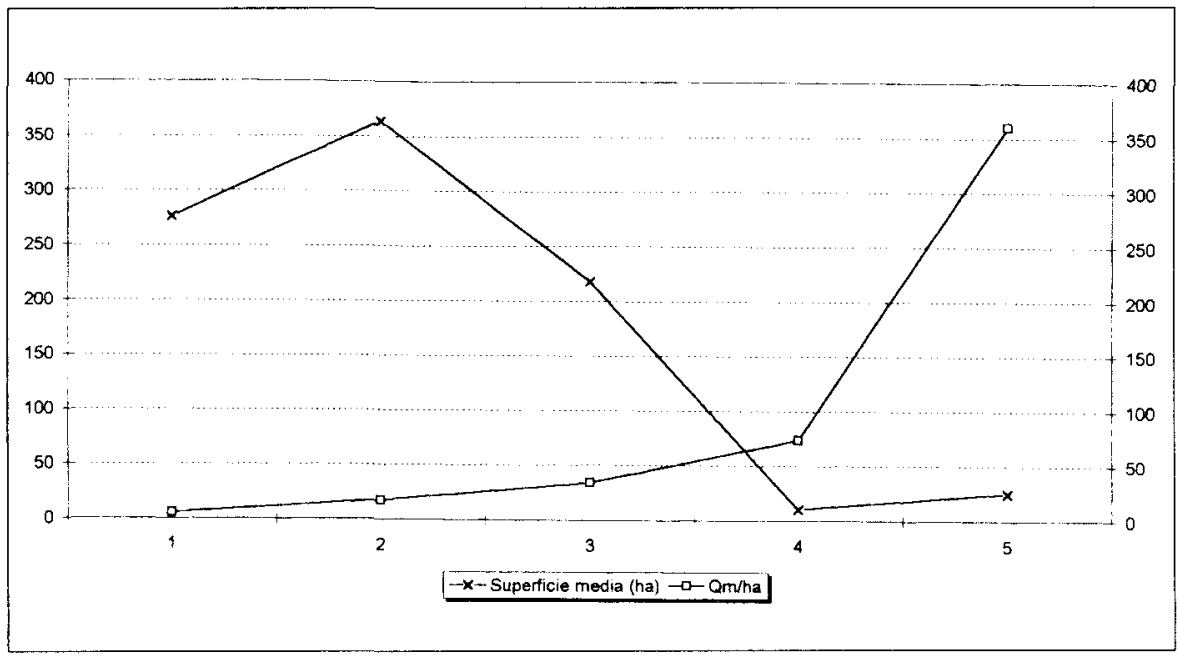

Fuente: AMJF, Leg. 1.119, Declaraciones...

altos. Deducir una interpretación correcta a partir de estos datos no es tarea fácil: $1^{\circ}$ ) Los aprovechamientos del monte jerezano, como vimos anteriormente, son muy variados, y no siempre es el corcho el producto principal, dado que en numerosas fincas los aprovechamientos ganaderos pueden ocupar el primer lugar; $2^{\circ}$ ) El estado de degradación del monte jerezano y la escasez de la masa arbolada; $3^{\circ}$ ) También cabe pensar que los propietarios privados tiendan a declarar unos rendimientos muy por debajo de los reales. El citado Medir Jofra estima entre el 20 y el 30 por 100 el nivel de ocultación en las declaraciones de los propietarios. De hecho, los rendimientos declarados, como vimos anteriormente, se encuentran un 25 por 100 por debajo de las estimaciones de Ceballos y Martín Bolaños. Esta situación no debe extrañarnos, si tenemos en cuenta la fase crítica en la que estos datos son solicitados a los propietarios - la fase final de la guerra civil y los primeros años de postguerra-. Finalmente, la propia variedad de los productos del monte jerezano puede hacer más fácil la ocultación de los datos de producción. Estudios posteriores deberán confirmar o no esta hipótesis.

Nos queda, en lo que al corcho se refiere, la comparación de las declaraciones de los propietarios privados con las cifras de producción del Ayuntamiento jerezano. Los montes de propios, la finca forestal de mayor dimensión del término de Jerez, al estar sujetos a ordenación, contaban 
con unos planes anuales de aprovechamiento. En la declaración del Ayuntamiento se expresan 8.400 quintales de corcho anuales, además de otros ingresos -4.000 metros cúbicos de leña, 4.000 arrobas de curtientes y 138.000 ptas. por el arriendo de pastos y montanera-.

Los montes de propios, con 6737 hectáreas declaradas que suponen el 41 por 100 de la superficie que rinde alguna producción de corcho, obtendrían 75.600 quintales en nueve años, esto es, el 34 por 100 de la producción total ${ }^{27}$. A partir de estas cifras, no podriamos considerar una mayor productividad o rendimiento medio por hectárea del monte municipal en relación con las fincas privadas. De dar por válidas las cifras municipales, la comparación, no demostraría, pues, que existieran indicios de ocultación en los propietarios privados. Pero también cabría pensar en una ocultación de la institución municipal. Al menos eso es lo que podría interpretarse si comparamos las cifras del plan veintenal de aprovechamientos del período $1908-1928{ }^{28}$ con las del año forestal 1921-22 ${ }^{29}$, que declara una producción de 21.716 quintales de udescorche de troncos y ramas", 2,5 veces más de lo proyectado para cada año. De ser así, los niveles de ocultación del Ayuntamiento jerezano serían incluso superiores a los de los propietarios privados ${ }^{30}$.

No debemos cerrar el tema de la producción de corcho sin reflejar al menos nuestras dudas respecto de los precios excepcionalmente bajos que declaran los informes municipales: poco más de tres pesetas el quintal métrico ${ }^{31}$, cuando Medir Jofra ofrece para el primer tercio del siglo cifras que oscilan entre las 15 y 20 pesetas de 1900 a una cotización situada entre 15

27 En principio, la cifra calculada por nosotros para nueve años podria considerarse aceptable, de tener en cuenta que en el pliego de condiciones para la subasta de los aprovechamientos de los montes de propios de 1908 se expresa la cifra de $170.297 \mathrm{qm}$. de corcho para el siguiente veintenio, lo que da una media anual de algo más de 8.500 qm. La información puede contrastarse en AMJF, sección Montes, Leg. 496, expediente 12.173 sobre antecedentes de la ordenación de los montes de propios.

28 Ibid., Ibid.

29 AMJF, sección Montes, Leg. 496, expediente 12.180, Plan de aprovechamiento y mejora del grupo de montes ordenados del término y propios de Jerez de la Frontera, año forestal 1921-1922.

30 Esta expresión debe entenderse como mera hipótesis de trabajo y refleja, simplemente, las dudas razonables que cualquier investigador debe plantearse. La economia forestal mediterránea es de una extraordinaria complejidad y la producción, de hecho, puede verse condicionada por causas imprevistas como son los incendios forestales. En el caso jerezano, tenemos referencias de dos graves incendios - todavia recordados por los guardas de montes más veteranos- que bien pudieron mermar la producción de corcho de los años posteriores: el primero, en 1915 (AMJF, Leg. 496, expediente 12.175, Policia Rural), y el segundo-del que he perdido las referencias-en 1929 (17 de abril), que afectó a cerca de 150 hectáreas de la Jarda y Charco de los Hurones.

31 AMJF, Montes, Leg. 496, expte. 12.180. 
y 25 pesetas -e incluso superiores - en los años 1926-29 ${ }^{32}$ para el quintal castellano de 46 kilos. Con razón, Sanz Fernández tiene la fundada impresión de que los ingenieros «infravaloraban el productos» y que «de ello se beneficiaban quienes concurrian a las subastas» ${ }^{33}$.

\section{B) Otros aprovechamientos del monte jerezano}

Al iniciar el estudio de los aprovechamientos del monte jerezano señalábamos que, con la excepción de la producción de corcho, los demás productos del monte estaban pésimamente especificados. Así, pese a la importancia que tienen los pastos y la montanera, las declaraciones no suelen indicar la carga ganadera de cada finca. Obviamente, los propietarios tienden a especificar exclusivamente los aprovechamientos forestales, por lo que el conocimiento de la carga ganadera en los montes deberá seguirse a través de otras fuentes, asunto que excede las limitaciones del presente trabajo.

Sin embargo, lo anterior no implica que debamos zanjar el tema, no sólo por la propia importancia ganadera de Jerez y la provincia de Cádiz, sino por las opiniones que sobre este tema vertían, en los años treinta del presente siglo, los ingenieros de montes Ceballos y Martín Bolaños, en su magnífico trabajo sobre el estado de la vegetación forestal de la citada provincia. Para estos autores, Cádiz es, desde antiguo, "una de nuestras más importantes regiones ganaderas" y cifran el número total de cabezas de ganado en torno a las $400.000{ }^{34}$. Ceballos y Martín Bolaños no juzgan necesario el conocimiento de tales cifras:

«...basta un somero recorrido por los montes, para apreciar desde el primer momento que se hallan sometidos a un pastoreo excesivo, falto de reglamentación y escaso de vigilancia" ${ }^{35}$.

32 Medir Jofra, R., op. cit., Alhambra, pp. 221 y 329.

Sanz Fernandez, J., op. cit., pp. 148-149.

"No conocemos datos precisos referentes al número total de cabezas de ganado que actualmente habitan la comarca, ni es posible fiarse de los que figuran en las estadisticas oficiales. siempre erróneas, a causa de las ocultaciones que los propietarios hacen de esta clase de riqueza; pero como suposición, que creemos aproximada, citaremos la cifra de 400.000 cabezas para la totalidad de la provincia...". Ceballos, L. y Martin Bolatios, M., op. cit., p. 83. Hemos de indicar que las estadisticas oficiales de la ganaderia española, publicadas recientemente por GEHR, muestran, para Cádiz, un total de 440.929 cabezas en el año 1933, distribuidas del siguiente modo: porcino, 25,5 por 100; caprino, 23 por 100; vacuno, 20,5; ovino, 19,9; caballar, 4,3; asnal, 3,5; y mular. 3,3. GEHR, op. cit, p. 350 . Nos basta señalar que los ingenieros de montes que estudiaron el monte gaditano se acercaron mucho a los datos reales de la carga ganadera de estos montes, pero también que las estadisticas oficiales, pese a sus deficiencias, no eran tan "erroneas".

35 Ceballos, L. y Martins Bolanos, M., op. cit., p. 83 
Estos autores no dudan en atribuir de un modo especial el lamentable estado de degradación del monte gaditano a la carga ganadera excesiva:

\begin{abstract}
«En los múltiples recorridos que nosotros hemos efectuado por los montes de la provincia, adquirimos la impresión de que el pastoreo abusivo en la mayoría de los casos, no sólo por efectuarse con un número de cabezas desproporcionado a la superficie de los pastizales y superior al debido, sino por la forma desordenada y falta de vigilancia con que el aprovechamiento se lleva a efecto; debiendo anotarse, además que, a pesar de la importancia que aquí tiene la ganaderia, no es frecuente entre los propietarios de montes preocuparse lo más mínimo de la conservación y mejora de los pastos, limitándose a efectuar de ellos una explotación rutinaria y abusiva, con lo cual trabajan, más o menos inconscientemente, por la degeneración simultánea de los montes y la ganadería" ${ }^{36}$.
\end{abstract}

Junto al corcho y los aprovechamientos de pastos y montanera, el siguiente aprovechamiento forestal imprescindible de los montes gaditanos es el de la leña y el carbón, fuentes de energia que no llegaron a ser sustituidas por el carbón mineral. Ceballos y Martín Bolaños atribuyen a la frecuencia y abuso de las cortas, podas y limpias de las fincas, junto al pastoreo excesivo, el estado de degeneración y agotamiento del monte, especialmente en los montes más cercanos a los grandes núcleos de población como Jerez ${ }^{37}$.

Las declaraciones forestales, sin embargo, no son lo suficientemente precisas en la declaración de las leñas para el carboneo, por lo que cabe pensar que, en este caso, el índice de ocultación era muy elevado, dado que el aprovecha miento se hacia sin la autorización del Ayuntamiento ni de la administración forestal ${ }^{38}$. Del total de propietarios, sólo diecinueve declaran alguna producción, cercana a los 3.000 quintales de carbón, mientras que los demás afirman cortar sólo lo necesario «para los gastos

36. Ibid., p. 87 .

37 Ibid., p. 80.

38. El tema de la corta de leña para el carboneo produjo un entrentamiento entre el jefe del Distrito Forestal gaditano y el alcalde de Jerez, del que da cuenta el Leg. 1.119. El servicio forestal advierte al Ayuntamiento de la existencia del decreto de 5 de octubre de 1938, citado por nosotros anteriormente, que obligaba a los propietarios a "solicitar autorización" de la administración Forestal para realizar los aprovechamientos de madera y leña, por lo que solicita al Ayuntamiento que "prohiba terminantemente la corta, saca o utilización de productos..." sin la correspondiente licencia. El alcalde jerezano pregunta al jefe del Distrito Forestal qué hace con aquellos trabajos comenzados antes del citado decreto, protesta por lo que considera un revés para «el abastecimiento normal de leñas y carbones" a la población, y señala, con razón - una "razón» muy empleada por los propietarios a lo largo del presente siglo-, que "en la relación de árboles consignada en el artículo primero del decreto no está incluido el acebuche... que aburda extraordinariamente en esta región". ANJF, Leg. op. 1119, Declaraciones... 
domésticos" o que es «imposible determinar previamente", y algunos, muy pocos, expresan la producción en estéreos —poco más de 200 estéreos-.

Salvo los citados, los demás aprovechamientos forestales son realmente marginales. Este es el caso de la madera: sólo seis propietarios declaran una producción insignificante, 4,1 toneladas, además de los que expresan haber obtenido madera para arados, palos e hincos.

\section{CONCLUSIÓN: BALANCE Y REFLEXIÓN SOBRE LA EVOLUCIÓN DEL MONTE JEREZANO}

Las Declaraciones forestales aportan numerosos datos para conocer el monte jerezano de los años cuarenta. También, y como sucede con toda fuente documental, al lado de las luces, hay que señalar sus sombras. Entre las primeras, hemos de destacar el conocimiento de la superficie del monte jerezano de la época, sin duda muy superior a la que mantiene en la actualidad. De las 55.000 hectáreas declaradas, el 40,7 por 100 tiene la consideración de arboladas, lo que viene a representar, de hacer caso a los datos de Ceballos y Martín Bolaños, el 16,5 por 100 de las masas arbóreas gaditanas y el 14,4 por 100 del término municipal.

Las declaraciones juradas también arrojan luz sobre la distribución de la propiedad del monte jerezano, repartido entre unas pocas familias de la élite local, que poseen la mayoría de la superficie -85 por $100-$. Ello da una dimensión media de la explotación muy elevada: más de 400 hectáreas de promedio, aunque buena parte de ellas se encuentran por encima de las 500 .

Como monte típicamente mediterráneo, la especie dominante en los montes de Jerez es el alcornoque, el cual forma diversas asociaciones con otras especies, destacando la que se produce junto al acebuche y el quejigo. Este tipo de monte determina, obviamente, los aprovechamientos que pueden obtenerse de él: pastos y montanera, corcho, leña y carbón.

El mejor aprovechamiento que puede estudiarse a partir de las declaraciones juradas es el del corcho, e incluso en ese caso hemos intentado hacerlo con las lógicas reservas. De todas formas, con unos rendimientos declarados a la baja, éstos suponen cerca de la cuarta parte de la producción provincial. Los demás aprovechamientos, aunque importantes por los testimonios de la época que hemos recogido, son imprecisos y constituyen la parte menos fiable de las declaraciones forestales. Si a ello añadimos que uno de los aprovechamientos esenciales del monte gaditano, los pastos y la montanera, no son considerados por los propietarios como 
específicamente forestales, nos encontramos ante un tema en el que las declaraciones arrojan escasa luz. En el caso de las leñas para el carboneo, la ocultación es patente, sobre todo si tenemos en cuenta que por las mismas fechas el servicio forestal gaditano advierte de la necesidad de solicitar autorización para la realización de las actividades forestales. En este caso, los propietarios contaban con un aliado eficaz: la propia ley, que no citaba al acebuche en la relación de especies protegidas ${ }^{39}$, siendo aquél, entre todas, la especie más estimada como combustible.

No deberíamos acabar este trabajo sin una reflexión, aunque breve, sobre dos aspectos que nos parecen esenciales: el proceso de degradación del monte jerezano y su relación con la coyuntura que atraviesa el monte español de la primera mitad del presente siglo, entre otras cosas porque podrian ayudarnos a comprender el difícil presente del monte mediterráneo, pese a la existencia de leyes protectoras, las cuales, no esta de más recordar, siempre han sido incumplidas.

El proceso de degradación del monte gaditano que proponen Ceballos y Martín Bolaños nos parece bastante aceptable en líneas generales, aunque hay que reconocer que se equivocaron en sus previsiones finales. Para estos autores ${ }^{40}$, el alcornoque es la especie dominante en nuestra provincia, entre otras razones, por ser la más resistente - lo que se explica por su corteza protectora - y la que mejor ha sabido adaptarse frente a la competencia. Otras ventajas del alcornoque respecto de las demás especies era su localización en las zonas menos habitadas de la provincia y las preferencias de los lugareños hacia el quejigo para la obtención de madera, el acebuche como combustible, y la encina para la montanera. De este modo, los enemigos del alcornoque serian, de un lado, la industria de curtidos, a la que ya nos hemos referido, floreciente en Ubrique y en otros pueblos de la provincia, y de otro, el proceso roturador que siguió a «nuestras funestas leyes desamortizadoras" ${ }^{41}$. El inicio de la industria corchera significaría, sin que éste fuera el propósito buscado, la aceleración de la

39 El tema del acebuche como especie que en Cádiz alcanza porte arbóreo es, por lo que vemos, bastante viejo: el hecho de que no se incluyera en las relación de especies autóctonas a proteger hasta 1988 nos da una idea de la protección adicional con que han contado los propietarios para llevar a efecto la roturación de los acebuchales con total impunidad, provocando la airada protesta de los ecologistas. Las causas ya la conocían Ceballos y Martín Bolaños en 1930: "...sus asociaciones son las más y en mayor grado perturbadas por el hombre, por ocupar los mejores suelos de la provincia para cultivo de cereales...", Ceballos, L. y Martin Bolaños, M., op. cit., p. 162.

40 Ibid., pp. 122-124

4 Ibid., lbid. 
destrucción del alcornocal, sobre todo por el uso y abuso del fuego para la mejora de los pastos, ya que al estar desprovisto de la corteza protectora, su resistencia disminuye. Posteriormente, ya a comienzos del presente siglo, los propietarios llegaron incluso a favorecer la dispersión del alcornoque frente al quejigo y otras especies, aunque con escaso éxito, dada la mala calidad del corcho en las zonas más húmedas, las preferidas del quejigo, y el mayor rendimiento en bellota de éste frente al alcornoque. Medir Jofra señala un elemento importante que pudo causar un daño notable a los alcornocales, como consecuencia de las transformaciones que se estaban produciendo en la demanda de la industria del corcho -incremento de la demanda del corcho para aglomerados, menos exigente en calidad que la industria taponera-:

«...se forzaron las sacas de corcho español en los años 1928 y 1929 Fue un mal general que perjudicó notablemente los montes alcornocales" ${ }^{42}$.

En este contexto, y pese a los problemas reseñados, Ceballos y Martín Bolaños auguraban unas previsiones buenas para el alcornoque, "considerando las rentas que proporcionan los alcornocales en nuestros días por el creciente valor de sus productos, y en especial el corcho y la bellota” ${ }^{43}$ :

\begin{abstract}
«...podemos asegurar que en líneas generales esta clase de montes tienden a aumentar su área, si bien la velocidad de esa restauración puede favorecerse en gran manera a pocas fuerzas que muevan sus propietarios. Los montes de Jerez, administrados bajo la inspección de un ingeniero municipal, en los pocos años de su gestión marcan ya el rumbo a seguir para los demás en esas y otras mejoras, siendo de esperar que su empleo cunda por toda la región en beneficio de sus intereses" ${ }^{44}$.
\end{abstract}

No cabe dudas de que se equivocaron estos autores. $Y$ el principal enemigo del alcornocal y del acebuchar no fue, precisamente, el que tanto denunciaron, el pastoreo excesivo, sino la roturación de estos terrenos y su posterior transformación agrícola. Pero para explicar este proceso es necesario que repasemos la coyuntura del monte español del momento, y cuál fue la respuesta del monte gaditano, al menos de los propietarios privados, que eran quienes poseian la mayoría de su superficie.

Durante el período estudiado, parte de los aprovechamientos del monte jerezano tenían una relativa importancia en el conjunto de la economía,

\footnotetext{
42 Medir Jofra, R., op. cit., p. 337.

43 Ceballos, L. y Martin Bolaños, M., op. cit., p. 124.

44 Ibid. Ibid
} 
como era el corcho y la ganadería, y otros eran imprescindibles para el abastecimiento de la población -leña y carbón- Sin embargo, a finales de este período Sanz Fernández observa cómo esos productos tradicionales - pastos, montanera, corcho, leña- pierden valor con respecto a las maderas y a las resinas ${ }^{45}$. En este sentido, la política forestal del franquismo no hizo sino reforzar y acelerar esta línea de actuación, favoreciendo a los sectores industriales de la madera, del aglomerado y de la celulosa, sectores ante los cuales poco podia hacer un monte como el gaditano, donde, además, la importantísima carga ganadera tal vez impedía una rápida adaptación.

Nos encontramos, pues, ante un período en el que se va a iniciar la marginación de los productos tradicionales del monte mediterráneo, su pérdida de valor en relación con los nuevos productos que demanda el mercado. En este proceso de «modernización» acelerada, el monte gaditano no participó o lo hizo en escasa medida, gracias a lo cual disponemos en la actualidad de una importantísima zona de vegetación forestal autóctona. Es algo que las generaciones actuales debemos agradecer: no siempre la «modernización» conduce al progreso o, para decirlo de otro modo, hay progresos que no nos llevan a ninguna parte.

Pero, cuando en los años cincuenta comience la crisis definitiva de la agricultura tradicional española, y dentro de ésta, del sector forestal tradicional -la dehesa-, muchos propietarios dispondrán de un escaso margen de maniobra y de adaptación. Y si a las dificultades económicas del monte .tradicional sumamos las exigencias modernizadoras de la administración leyes de fincas manifiestamente mejorables, Reforma Agraria de Andalucía, etc.-, no era difícil comprender el destino de estos frágiles terrenos: su transformación agraria, aunque nunca hubieran tenido esa vocación.

${ }^{45}$ Sanz Fernandez, J., op. cit., pp. 150-151. 